\title{
Gastrointestinal Stromal Tumors with Unusual Presentations: Case Series
}

\author{
Mohamed M Refaey ${ }^{1}$, Mohamed H Emara ${ }^{1}$, \\ Tarik I Zaher ${ }^{1}$, Tarik M Ezzat ${ }^{2}$, \\ Magdy B El-Moghazy ${ }^{3}$ \\ 1 Tropical Medicine Department, Faculty of Medicine, Zagazig University, Zagazig, 44519, \\ Egypt. \\ 2 General Surgery Department, Faculty of Medicine, Zagazig University, Zagazig, 44519, \\ Egypt. \\ 3 General Surgery Department, Faculty of Medicine, Mansoura University, Mansoura 35516, \\ Egypt.
}

Corresponding Author Mohamed M Refaey Mobile:

+201000089159

E mail:

rifae20007@yahoo.co

$m$

Received :12/6/2012

Accepted after revision: $13 / 7 / 2012$

Key words:

Gastrointestinal

stromal tumors,

hematemesis, melena,

abdominal mass,

immuonohisto-

chemisrty.
Background: Gastrointestinal stromal tumors are rare mesenchymal tumors; and it may be presented by a variety of manifestations..

Case Presentation: A 56-year-old Egyptian male patient presented with a massive gastrointestinal bleeding with hemodynamic instability, with history of previous two mild attacks. After initial resuscitation, endoscopic, pathological and immunohistochemical examination; diagnosis of gastrointestinal stromal tumor was confirmed. The patient's tumor is of low risk and complete surgical excision with safety margins was performed. Another 60-year-old Egyptian female diabetic patient presented for preoperative assessment for cataract operation. On examination large abdominal mass was discovered. After initial diagnostic work up surgical resection was done. Pathological and immunohistochemical examination confirmed the diagnosis of gastrointestinal stromal tumor.

Conclusion: Gastrointestinal stromal tumors, should not be overlooked while investigating cases of massive upper gastrointestinal bleeding and cases of large abdominal masses especially in elderly patients.

\section{INTRODUCTION}

Gastrointestinal stromal tumors (GISTs) are mesenchymal tumors specific for the gastrointestinal tract (GIT) and are rare, representing 0.1$3 \%$ of all GIT cancers with an estimated incidence of 15 per million. Gastrointestinal tract bleeding (50\%) is the most common presentation, followed by abdominal pain (20$50 \%)$ obstruction (20\%) and approximately one third are detected incidentally [1]. GISTs initially presenting as an abdominal mass are exceedingly rare [2]. From 10 to $30 \%$ of them are malignant and show liver metastases or intra-abdominal spread at the time of diagnosis [3].

GISTs can be a cause of obscure GIT hemorrage and should be kept in mind when conventional investigations such as esophagogastroduodenoscopy (EGDS) and colonoscopy fail to detect bleeding lesions, especially when located in the small bowel [3].

The digestive hemorrhage occurs if they erode or ulcerate through the bowel mucosa they can cause bleeding that is usually intermittent, but massive bleeding is uncommon $[4,5]$.

Diagnosing these lesions is difficult because they tend to be inaccessible to routine diagnostic methods. Variable sensitivities and specificities for diagnosing GISTs are found and includes EGDS, barium studies, selective visceral angiography, wireless capsule endoscopy, radioactive isotope bleeding scans, CT scans, MRI and exploratory laparotomy is often the last option $[5,6]$.

We recorded a case with recurrent attacks of hematemesis and melena admitted due to a massive upper GIT 
bleeding and a case with accidentally discovered large abdominal mass originating from the transverse colon proved to be GIST to raise the awareness among clinicians about the uncommon presentation of these tumours.

\section{Case 1}

\section{History}

A 56-year old rural male patient presented by a massive attack of hematesis and melena with no history of chronic medical disease. On detailed history taking he gave history of 2 previous mild attacks of hematesis and melena.

\section{Examination}

At admission the patient was extremely pale, blood pressure 80/40, pulse 126 with regular rhythm, otherwise free.

\section{Investigations and management}

Initial resuscitation measures were done (2 wide pore canuulae were inserted, fresh blood transfusion, colloids and crystalloids infusion). Full laboratory investigations were done including complete blood counts, liver function tests, kidney function tests, coagulation profile and all were free apart from severe normocytic anemia of $6.5 \mathrm{gm} \%$. Decision to examine the patient by EGDS was taken.

\section{Endoscopic findings}

a- Pale mucosa, small hiatus hernia and fungating corpus cauliflower mass easily bleed on touch with luminal encroachment, multiple punch biopsies were taken for histopathology (Figure 1).

b- Pathological examination of endoscopic biopsies revealed pieces of tumor tissue formed of oval and spindle cells with vesicular nuclei and high mitotic index, picture highly suggestive of GIST for immunohistochemistry (Figure 2).

\section{Immunohistochemistry}

Sections were prepared from paraffin block, processed in Benchmark XT (Ventana) and stained by monoclonal antibodies, using $\mathrm{DAB}$ as chromogen and hematoxylin as counter-stain. The sections were treated against C. kit (CD117) and $\mathrm{CD} 34$ and tumor cells were strongly positive for it (Figure 3).

\section{Surgical Resection}

Surgical resection of a large wedge of the stomach $10 \times 14 \mathrm{~cm}$ including the tumor with safety margins was done.

\section{Pathological examination}

Pathological examination of the resected part of the stomach revealed: non capsulated tumor mass of $3.5 \times 2.5 \times 1 \mathrm{~cm}$. Sections prepared showed tumor tissue formed of spindle cells. Mitotic activity was 2-3/ 50 high power field (HPF), with congested vessels and ulcerated mucosal covering. Sections prepared from the surrounding gastric wall showed free surgical margins.

\section{Case 2}

\section{History}

A 60-year old female patient with type 2 diabetes presented for preoperative assessment for cataract operation with no other manifestations.

\section{Examination}

On examination the patient was generally good, blood pressure 130/70, pulse 80 with regular rhythm, large firm mildly tender, limited mobility mass felt in left lumbar region with normal overlying skin. She denies nausea, vomiting, weight loss or bowel habits disturbance.

\section{Investigations and management}

1- Abdominal ultrasound showed ill defined mass related to the colon

2- Abdominal CT scans showed large heterogeneous mass 9x6 cm inseparable from the colon and extending to the abdominal wall (Figure 4).

3- Laboratory investigations: liver functions, kidney functions, complete blood counts showed anemia with hemoglobin level of $10.2 \mathrm{gm} \%$.

4- Surgical Resection: On laparotomy the mass was originated from the transverse colon and attached to the lateral abdominal wall. Surgical resection of the mass and related colon with safety margin and re-anastmosis was done. 


\section{Pathological examination:}

Pathological examination of the resected part revealed: non capsulated tumor mass $8 \times 7 \times 4 \mathrm{~cm}$. Sections prepared showed tumor tissue formed of spindle cells intermingled with mixed inflammatory cell infiltrate. The growth involved colonic wall. Cut colonic margins were free. Two dissected lymph nodes were free from the tumor and showed reactive hyperplesia. Mitotic activity was 2-3/ $50 \mathrm{HPF}$.

\section{Immunohistochemistry}

Sections were prepared from paraffin block, processed in Benchmark XT (Ventana) and stained by monoclonal antibodies, using $\mathrm{DAB}$ as chromogen and hematoxylin as counter-stain. The sections were treated against C. kit (CD117) and CD34 and tumor cells were strongly positive for both.

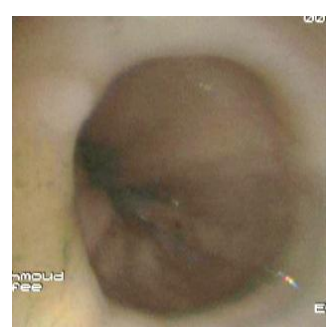

(a)

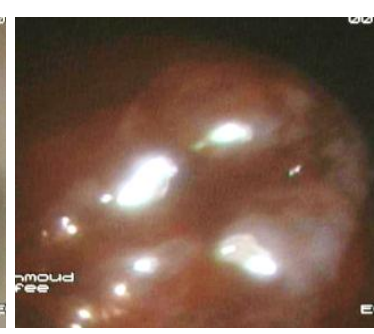

(b)

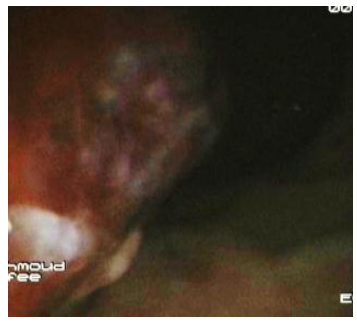

(c)

Figure (1): Endoscopic findings; (a) sliding hiatus hernia with pale esophageal mucosa, (b) large fundal mass, (c) ulcerated surface of the mass

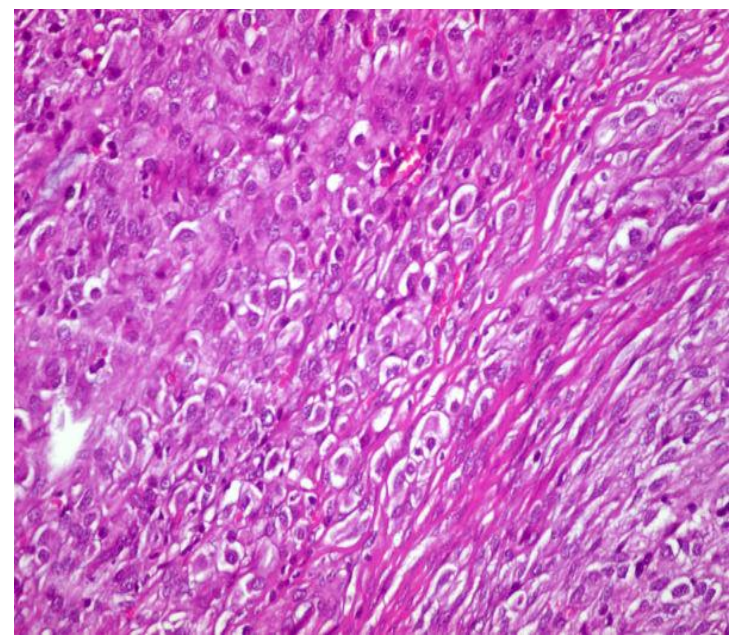

Figure (2): Pathological sections from the tumor showing spindle shaped cells with high mitosis

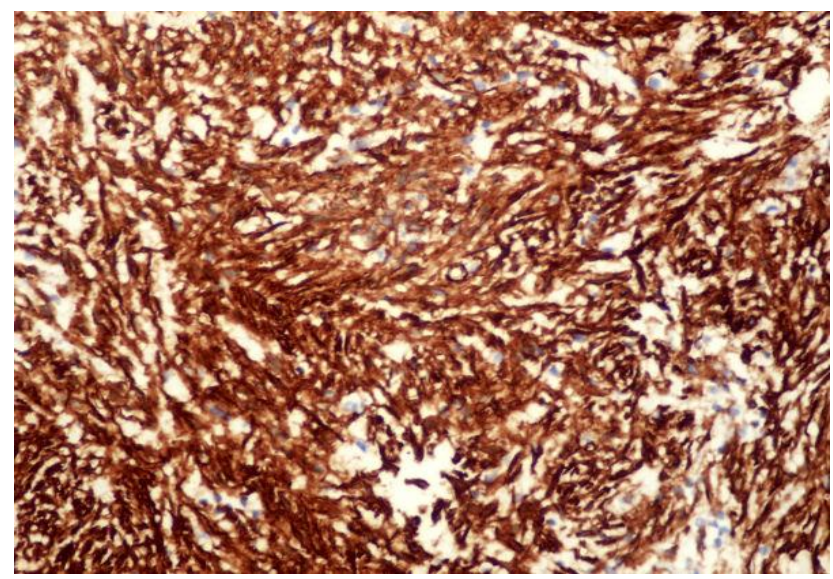

Figure(3): Immunohistochemistry showing the tumor positivity for CD 117 


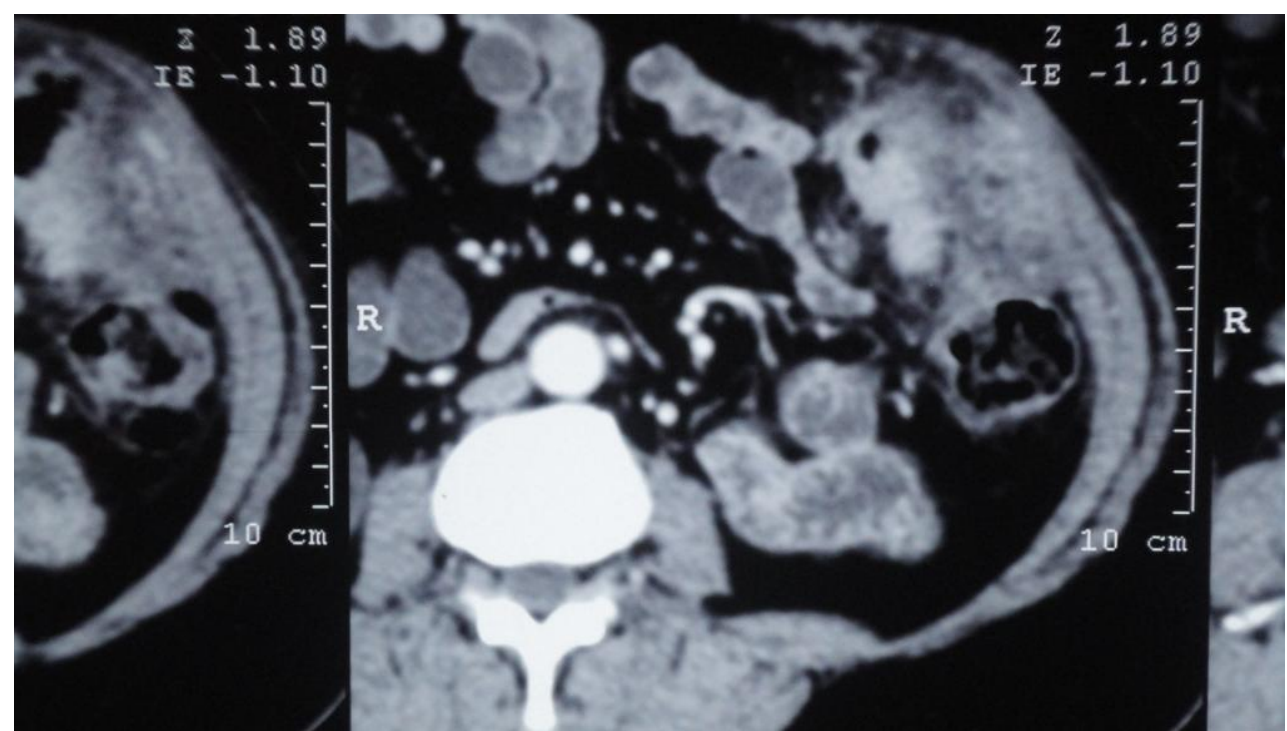

Figure (4): CT scan shows the mass related to colon and abdominal wall.

\section{DISCUSSION}

It is now believed that GIST tumors arise either from stem cells that differentiate towards interstitial cells of Cajal (these cells form part of the myenteric plexus in the GIT and regulate peristalsis i.e. link the autonomic innervations of the gut with smooth muscle cells and regulate GIT motility) or directly from interstitial cells of Cajal and not from smooth muscle cells [7]. GIST usually seen in adults in the sixth decade of life [8] and this coincide with the findings of our cases.

These tumors grow intraluminally or extraluminally. When the growth pattern is extraluminal, patients may be symptom free for a long time and present with very large exoluminal masses [8], this is obvious in our second case where the patient presented by huge intraabdominal mass that was accidentally discovered while examined for preoperative assessment. When it grew intraluminally manifestations develop, and hence our first case experienced recurrent attacks of bleeding inspite of the small size of the resected tumor due to erosions into the covering mucosa and the surrounding vessels.

In Egypt variceal bleeding is the most common cause of upper GIT bleeding [9], and GIST represent a very uncommon cause in comparison to variceal bleeding and that is why it is not usually thought when investigating cases of upper GIT bleeding in our community.

GIST is an unusual cause of upper GIT bleeding, and has a high likelihood to rebleed and this explains the recurrent attacks of bleeding reported in the first case. These bleeding tumors need to be investigated urgently as an inpatient rather than as an outpatient [10]. This is obvious in our case, the patient was admitted due to a massive GIT bleeding, and that is why urgent resuscitation was performed hand in hand with the battery of investigations performed. Early surgical intervention, either open or laparoscopic resection, is the treatment of choice to prevent re-bleeding [10], in the 12 months follow up period after successful surgical resection our case had no attacks of bleeding and no additional morphological changes on endoscopic examination.

The second case presented here mimicked other abdominal conditions like colon cancer, mesenteric mass, stomach cancer, ovarian cancer and retroperitoneal tumor both clinically and radiologically. Although there is no specific CT findings for GIST tumors, we performed a contrast-enhanced CT scan because it is the imaging modality of choice for patients with suspected abdominal mass, as it helps in both pre-operative staging and to evaluate for metastatic disease [11]. Preoperative biopsy carries a risk of hemorrhage due to the friable nature of these tumors [2], and hence we avoided it because we planned for a definitive surgery.

Kit protein is a tyrosine kinase growth factor receptor present in $90 \%$ of GIST cells. The incidence of GISTs has increased in the last few years due to better detection as all mesencymal tumours are now being tested for CD117. CD117 
(Kit protein) is the product of c-kit protooncogene, located on chromosome 4q11-21 [12]. Our cases tested positive for CD117 by immunohistochemistry and this leaves no doubt that it is a case of GIST.

Complete surgical resection is accomplished in $40-60 \%$ of all GIST patients, and in $>70 \%$ of those with primary non-metastatic GIST [10]. In our cases we had completely excised the tumor, and this was confirmed by the safety margin on pathological examination.

GISTs exhibit a highly variable behavior after resection of the primary tumor. These patients need to be followed up on a long term basis as local recurrence and metastases can occur many years after surgery. These tumors spread by the haematogenous route predominantly to the liver. Lymph node involvement is very rare and therefore lymphadenectomy is not routinely indicated [13]. In the first case no extragastric spread was reported at the time of diagnosis nor in the 12 months follow up period. While in the second case no intraluminal nor extraluminal recurrence was reported in the 12 months of follow up.

Local recurrence or metastases develop in approximately $50 \%$ of patients who had potentially curative operation [14]. The two most important tumor factors for local recurrence and metastasis are tumor size and mitotic rate (size $>5 \mathrm{~cm}$ and mitosis $>5$ per $50 \mathrm{HPF}$ increases the risk). Other prognostic factors are completeness of resection, age, and tumor location. Gastric GISTs have a lower risk of tumor recurrence than esophageal, small bowel or large bowel GISTs [15].

The first case seemed to be a low risk case and this is directly related to the small size of the tumor $<5 \mathrm{~cm}$, low mitotic index (2-3/50 HPF) and complete excision of the tumor with free safety margins; this explains the good general condition and absence of rebleeding after surgical resection through the follow up period.

The second case seemed to be an intermediate risk; large size of the tumor $(>5 \mathrm{~cm})$ is a high risk parameter contrary to the low mitotic index (2$3 / 50 \mathrm{HPF}$ ) and complete excision of the tumor with free safety margins. Similar controversy in the correlation between tumor size and mitotic index was also reported by Patil et al. [2].

The median disease survival for patients with primary GIST is approximately 5 years [16].
This is not elucidated in these cases because we followed the patients for 12 months only.

Imatinib (a tyrosine kinase inhbitor) is approved for the treatment of advanced disease. The recommended starting dose is $400-600 \mathrm{mg}$, this drug is safe and effective [17]. Because the tumors were completely resected, low risk of resected tumors; this drug was not given and these cases were candidates for regular follow up.

\section{CONCLUSION}

Gastrointestinal stromal tumors, should be kept in mind while investigating cases of massive upper gastrointestinal bleeding and cases of large abdominal masses especially in elderly patients.

Acknowledgements: The authors would thank Dr Emad Abd El-Hamed for helping in preparation of the figures of this case

Funding: Non .

Conflicts of interest: The authors declare that they have no conflict of interest.

Ethical approval: Not needed.

\section{REFERENCES}

1. Rossi CR, Mocellin S, Mancarelli R, Foletto M, Pilati P, Nitti D et al. Gastrointestinal stromal tumours: from a surgical to a molecular approach. Int J Cancer 2003; 107:171-176.

2. Patil S, Jain S, Kaza RC, Chamberlain RS. Giant gastrointestinal stromal tumor presenting as a palpable abdominal mass: an unusual presentation ISRN Surg $2011 ; 2011: 894829$.

3. Miettinen M, Sarlomo-Rikala M, Lasota J. Gastrointestinal stromal tumors: recent advances in understanding of their biology. Hum Pathol1999; 30(10):1213-1220.

4. Vilallonga $\mathrm{R}$, Sanchez JL, Armengol $\mathrm{M}$. Gastrointestinal Stromal Tumor: Cause of Gastrointestinal Bleeding. Gastroenterol Res 2010; 39(2): 93-95

5. Lahoti S, Fukami N . The small bowel as a source of gastrointestinal blood loss. Curr Gastroenterol Rep 1999;1(5):424-430.

6. Serio G, Montresor E, Costantini E, Maragos S, Bortolasi L, Nicoli $\mathrm{N}$ et al .Stromal tumors of the stomach. Our experience with 25 patients. Chir Ital 1998;50(5-6):35-40.

7. Gay G, Delvaux M, Fassler I . Outcome of capsule endoscopy in determining indication and route for push-and-pull enteroscopy. Endoscopy 2006; 38(1):49-58. 
8. Kindblom LG, Remotti HE, Alderberg F, MeisKindblom JM . Gastrointestinal Pacemaker Cell tumor (GIPACT) GIST show phenotypic characteristics of the Interstitial cell of Cajal. Am J Patho1998; 152.

9. Rai C, Hota C, Ganguli L, Mallick C . Gastric Stromal Tumour presenting as Upper Gastrointestinal Bleed. MJAFI 2008 ; 64 : 171-172

10. Singhal T, Doddi S, Leake T, Parsi S, Hussain A, Chandra $\mathrm{A}$ et al .Upper gastrointestinal bleeding due to gastric stromal tumour: a case report. Cases J 2010 ;12, 3:58

11. Da RT, Modesto A, Bazzocchi M . Gastrointestinal stromal tumour: spiral computed tomography features and pathologic correlation. Radiol Med 2006;111:661-673.

12. Gado AS, Ebeid BA, Abdelmohsen AM, Axon AT . Clinical outcome of acute upper gastrointestinal hemorrhage among patients admitted to a government hospital in Egypt. Saudi J Gastroenterol 2012;18:34-9
13. Demetrie GD, Lewis JJ, Leung D, Mudan SS, Woodruff JM, Brennan MF. Two hundred GIST; Recurrence patterns and prognostic factors for survival. Ann Surg 2000; 231:51-58.

14. Woodall CE III, Brock GN, Byam JA, Scoggins CR, McMasters KM, Martin RC II . An evaluation of 2537 gastrointestinal stromal tumors for a proposed clinical staging system. Arch Surg 2009; 144(7):670-678.

15. Ng EH, Pollock RE, Romsdahl MM: Prognostic implications of patterns of failure for gastrointestinal leiomyosarcomas. Cancer 1992; 69:1334-1341.

16. Josnsuu H, Fletcher C, Dimitrijevic S, Silberman S, Roberts P, Demetri G . Management of malignant GIST. Lancet Oncol 2002; 3:655-664.

17. Benjamin RS, Blanke CD, Blay JY, Bonvalot S, Eisenberg B . Management of Gastrointestinal stromal tumors in the Imatinib era. Oncologist $2006 ; 11: 9-20$. 